\title{
The contribution of cholinergic and dopaminergic afferents in the rat prefrontal cortex to learning, memory, and attention
}

\author{
MICHAEL E. RAGOZZINO \\ University of Ilinois, Chicago, Illinois
}

\begin{abstract}
Recent experiments have provided valuable information about the functional organization of the subregions of the rat prefrontal cortex. This review assesses the findings from studies in which the role of the cholinergic and dopaminergic systems in the anterior cingulate, prelimbic, and infralimbic areas was investigated by using several different approaches - that is, chemical lesions, pharmacological manipulations, or in vivo microdialysis. Cholinergic and dopaminergic input to the prelimbic and infralimbic subregions may modulate multiple behavioral processes. Activation of the muscarinic cholinergic receptors or activation of the dopamine $D_{1}$ receptors in the prelimbic and infralimbic areas modulates working memory processes. Cholinergic input to the prelimbic and infralimbic areas may also modulate attention, since changes in acetylcholine release occur when there is an increase in attentional demands. In addition, activation of dopaminergic receptors in the prelimbic and infralimbic areas influences behavioral flexibility. Cholinergic input to the anterior cingulate does not appear critical for working memory but likely contributes to attentional processes. Overall, the results suggest that acetylcholine and dopamine affect multiple behavioral functions subserved by the rat prefrontal cortex that depend on modulating activity in specific prefrontal cortex subregions.
\end{abstract}

The human prefrontal cortex is thought to be a region of the cerebral cortex that is intimately involved in some of the most complex behavioral and cognitive functions (Fuster, 1997; Shallice \& Burgess, 1991; Stuss \& Benson, 1986). Neuropsychological assessments of prefrontaldamaged patients commonly reveal dysfunctions in affect, attention, working memory, strategy selection, and temporal sequencing (Burgess \& Shallice, 1996; Chao \& Knight, 1995; Damasio, Tranel, \& Damasio, 1990; Freedman, Black, Ebert, \& Binns, 1998; Kesner, Hopkins, \& Fineman, 1994; McDowell, Whyte, \& D'Esposito, 1998; Milner, Petrides, \& Smith, 1985; Owen, 1997; Owen, Morris, Sahakian, Polkey, \& Robbins, 1996; Swain, Polkey, Bullock, \& Morris, 1998). Largely because the human prefrontal cortex is proposed to be a brain region involved in high-level cognitive processes, attempts to understand the neural mechanisms underlying these processes have involved experiments carried out predominantly in nonhuman primates. Nevertheless, all mammalian species appear to have a prefrontal cortex, although there is still debate on how to anatomically define the prefrontal cortex in some species (Preuss, 1995; Uylings \& van Eden, 1990). In addition, during recent decades, there have been numerous experiments in rodents that suggest that this species can serve as a useful model for understanding prefrontal

Correspondence concerning this article should be addressed to $\mathrm{M}$. $\mathrm{E}$. Ragozzino, Department of Psychology, University of Illinois at Chicago, 1007 W. Harrison St., Chicago, IL 60607 (e-mail: mrago@ uic.edu). cortex functioning (Kolb, 1984, 1990). In particular, pharmacological manipulations and lesions of the rat prefrontal cortex lead to behavioral deficits comparable with those found in humans with prefrontal cortex damagethat is, impairments in working memory, strategy selection, and temporal sequencing (Brito \& Brito, 1990; de Bruin, Swinkels, \& de Brabander, 1997; DeCoteau, Kesner, \& Williams, 1997; Frysztak \& Neafsey, 1994; Gallagher, McMahan, \& Schoenbaum, 1999; Granon \& Poucet, 1995; Kesner \& Holbrook, 1987; Kesner, Hunt, Williams, \& Long, 1996; Kolb, Burhmann, McDonald, \& Sutherland, 1994; Muir, Everitt, \& Robbins, 1996; Ragozzino, Adams, \& Kesner, 1998; Ragozzino, Detrick, \& Kesner, 1999a; Ragozzino \& Kesner, 1998; Ragozzino, Wilcox, Raso, \& Kesner, 1999).

Another similarity in the experimental findings among different mammalian species is that separate prefrontal cortex subregions differentially contribute to various behavioral and cognitive functions (Bussey, Muir, Everitt, \& Robbins, 1997; DeCoteau et al., 1997; Dias, Robbins, \& Roberts, 1996; Eichenbaum, Clegg, \& Feeley, 1983; Fuster, 1997; Kesner et al., 1996; Mogensen \& Holm, 1994; Petrides, Alivisatos, Evans, \& Meyer, 1993; Ragozzino et al., 1998; Ragozzino, Detrick, \& Kesner, 1999a; Seamans, Floresco, \& Phillips, 1995; Wilson, Scalaidhe, \& Goldman-Rakic, 1993). However, an understanding of the mechanisms in different prefrontal cortex subregions that underlie various functions remains incomplete. In recent years, there has been an interest in determining the neurochemical changes in different parts of the prefrontal cortex that influence various behavioral processes - that 
is, learning and attention. These experiments have involved the use of different techniques, such as intracranial drug injections, specific neurotoxic lesions, and in vivo microdialysis. Although the rat prefrontal cortex consists of a medial and a lateral sector, the vast majority of these studies have concentrated on the neurochemical mechanisms in the medial prefrontal cortex-particularly the anterior cingulate, prelimbic, and infralimbic subregions - that underlie particular cognitive processes. This review focuses on cholinergic and dopaminergic actions in the rat anterior cingulate, prelimbic, and infralimbic subregions as they relate to different behavioral processes involving attention, learning, and memory. The discussion will be limited to studies directly measuring or manipulating dopamine and acetylcholine changes in these prefrontal cortex subregions. In some of the experiments discussed, the role of these neurochemicals in only the prelimbic area was examined, whereas in other investigations, their role in the prelimbic and infralimbic areas combined was examined. These findings are discussed under one heading, but whether a particular study involved only the prelimbic region or both the prelimbic and the infralimbic areas is duly noted.

\section{ANATOMY}

The rat prefrontal cortex is a heterogenous structure that consists of anatomically distinct subregions that may also represent separate functional compartments. The prefrontal cortex is commonly defined by either reciprocal or comparatively dense connections with the mediodorsal thalamic nucleus, relative to other thalamic nuclei (Groenewegen, 1988; Uylings \& van Eden, 1990). The connections between the mediodorsal thalamic nucleus and the prefrontal cortex are topographically organized, leading to the following two subdivisions of the rat prefrontal cortex: (1) a medial division consisting of the medial precentral area, the dorsal and ventral anterior cingulate, and prelimbic, infralimbic, and medial orbital areas and (2) a lateral division encompassing the lateral orbital cortex and the dorsal and ventral agranular insular area (Groenewegen, 1988; Krettek \& Price, 1977; Leonard, 1969; Uylings \& van Eden, 1990).

The main source of cholinergic afferents to the rat prefrontal cortex is the basal forebrain (Gaykema, Luiten, Nyakas, \& Traber, 1990; Woolf, 1991). In the basal forebrain, the nucleus basalis magnocellularis and the horizontal limb of the diagonal band area are the principal regions that send cholinergic projections to the prefrontal cortex (Gaykema et al., 1990; Woolf, 1991). The vertical diagonal band and medial septal area appear to have a sparse cholinergic projection to the prefrontal cortex (Gaykema et al., 1990). Furthermore, the laterodorsal tegmental nucleus also has cholinergic projections to the ventromedial prefrontal cortex (Satoh \& Fibiger, 1986).

The dopaminergic afferents to the rat prefrontal cortex originate from the midbrain tegmentum (Berger, Gaspar, $\&$ Verney, 1991). The dopamine terminals in the ventral portions of the medial prefrontal cortex originate from the ventral tegmental area and terminate predominantly in layers V and VI (Berger et al., 1991). The dopamine projections to the anterior cingulate originate mainly from the substantia nigra and terminate in the superficial layers (Berger et al., 1991).

\section{THE ROLE OF CHOLINERGIC TRANSMISSION IN THE PREFRONTAL CORTEX ON COGNITIVE PROCESSES}

Most experiments that have focused on how cholinergic transmission in the medial prefrontal cortex influences behavioral and cognitive processes have employed working memory and attention tasks. In addition to traditional pharmacological approaches, in vivo microdialysis and the selective cholinergic neurotoxin 192 IgG-saporin are now being employed to facilitate an understanding of how cholinergic transmission in separate brain regions influences different behavioral and cognitive processes. This section will include some of the most recent experiments that have used these techniques.

\section{Cholinergic Influences in the Prelimbic and Infralimbic Areas on Working Memory}

The findings from several studies suggest that the rat prefrontal cortex, like the nonhuman primate and human prefrontal cortices, is involved in working memory (DeCoteau et al., 1997; Delatour \& Gisquet-Verrier, 1996; Granon, Poucet, Thinus-Blanc, Changeux, \& Vidal, 1995; Kesner et al., 1996; Kolb et al., 1994; Ragozzino et al., 1998; Seamans et al., 1995). Working memory commonly refers to the short-term storage of information that enables accurate execution of a response. Spatial delayed alternation and delayed nonmatching-to-sample tasks are frequently used to assess working memory processes. In the spatial delayed alternation task, a rat is often tested in a T-maze in which its choice of which arm to enter is always between the same two arms. A rat is reinforced for choosing either arm on the first trial, and on each subsequent trial, it must alternate and always choose the opposite arm to that chosen on the previous trial to receive a reinforcement (or avoid an aversive stimulus - i.e., a shock). A delay is inserted between trials, during which the rat must remember which arm it had just entered on a trial.

In contrast to the spatial delayed alternation task, each trial in a delayed nonmatching-to-sample task is usually divided into a sample phase and a test phase. In the sample phase, a stimulus is presented that the subject must respond to. After a delay, the test phase begins, in which two stimuli are presented-one that is identical to the sample phase and one that is novel for that trial. The novel stimulus must be chosen to receive a reinforcement. In order to choose the novel stimulus, the stimulus that was presented during the sample phase has to be remembered.

Although the spatial delayed alternation and the delayed nonmatching-to-sample tasks have a working mem- 
ory component to them, these tasks also take advantage of rats' natural tendency to alternate (Dember \& Fowler, 1958; Olton \& Schlosberg, 1978). Therefore, the tasks also have a strategic component to them, in which rats have a bias to choose the location that has been least recently visited or the novel stimulus. This raises the possibility that a deficit in one of these tasks may result from an impairment in proper execution of an alternation strategy, as opposed to a working memory deficit. If a deficit in these tasks is due to a selective impairment in using a strategy, the deficit should occur independent of the delay. However, if a particular manipulation impairs performance only at long delays, but not at short delays, these findings would suggest that the impairment is likely due to a working memory deficit. Alternatively, a manipulation may impair both strategy selection and working memory. Because of the strategic component to the task, systematically examining the effects of different delays in a spatial delayed alternation or a delayed nonmatching-to-sample task can better elucidate whether a manipulation selectively impairs working memory.

To determine whether cholinergic transmission in the prefrontal cortex influences working memory, most studies have used intracranial drug infusions. Brito and colleagues (Brito, Silva, \& Brito, 1989) demonstrated that injections of the muscarinic cholinergic antagonist scopolamine into the prelimbic area impaired performance in a T-maze, using a delayed nonmatching-tosample procedure. Although the scopolamine infusions impaired performance, the effects of scopolamine were not examined across different delays, opening up the possibility that blockade of muscarinic receptors in the prelimbic area impaired the ability to use a delayed nonmatchingto-sample strategy, as opposed to a selective deficit in working memory. In a more recent experiment, blockade of muscarinic cholinergic transmission in the prelimbic area was found to impair both delayed nonmatching-tosample and matching-to-sample in a spatial memory test using a 30-sec delay between the sample phase and the test phase (Granon et al., 1995). In this study, the effects of muscarinic cholinergic blockade was not examined at different delays either. However, the authors did report that pilot results indicated that prelimbic inactivation with the local anesthetic lidocaine did impair performance at a 30-sec delay, but not at a 5-sec delay (Granon et al., 1995). Because of the delay-dependent effects with lidocaine infusions, the impairment following scopolamine infusions into the prelimbic area may be due to a working memory deficit.

To better determine whether muscarinic cholinergic transmission in the prelimbic and infralimbic areas influences working memory processes, the effect of scopolamine infusions into the prelimbic-infralimbic areas across different delays was investigated recently in our laboratory with an experiment that used a spatial continuous recognition procedure (Ragozzino \& Kesner, 1998). A continuous recognition procedure was developed in order to use a test that was comparable with the contin- uous recognition procedures employed to study working memory in humans. In this task, rats were exposed sequentially to 12 arms during a session. Three or 4 of the arms were presented for a second time in a session. A rat learned that a cereal reinforcement was at the end of the arm during the first presentation, but not during the second presentation. The number of intervening arm choices between the first and the second presentations of an arm varied from zero to six. Memory was assessed by the latency to enter an arm during the second presentation. Rats remember entering a location during a session and thus avoid entering the location during the second presentation. With an increase in lag, there is an increase in the delay and interference between the first and the second presentations of a spatial location; thus, latencies decrease with longer lags. Scopolamine injections produced a doseand lag-dependent impairment in spatial working memory. More specifically, blockade of muscarinic receptors in the prelimbic-infralimbic areas did not impair memory at the shortest lag ( $\operatorname{Lag} 0$ ) but did impair performance with increased lags. Furthermore, the results also indicated that the deficit following scopolamine infusions increased at longer lags. The lag-dependent deficit is consistent with a working memory impairment, since scopolamine infusions into the prelimbic-infralimbic areas impaired spatial memory only at longer lags, when temporal and spatial interference was greatest. Moreover, the scopolamine-induced impairment was reversed with combined intracranial infusions of oxotremorine, a muscarinic agonist, suggesting that the impairment was specifically produced by blockade of muscarinic receptors in the prelimbic-infralimbic region. Overall, a continuous recognition procedure, such as that used in this study, has advantages in assessing memory performance across several different delays and levels of interference and may provide a useful paradigm for understanding the neurochemical mechanisms that underlie working memory.

In addition to spatial working memory tests in a T-maze or a radial arm maze, the effects of cholinergic drugs into the prelimbic area on matching-to-position tests in operant chambers have been investigated in other experiments (Aura \& Riekkinen, 1999; Broersen, Heinsbroek, de Bruin, Uylings, \& Olivier, 1995). In these tasks, rats were presented with one bar to press in a sample phase and then, after a delay, were presented with two bars in the test phase. Depending on the procedure in the test phase, a rat learned to press the bar that was the same as (matchingto-position) or the bar that was opposite to (nonmatchingto-position) that in the sample phase. Although this task has a spatial memory component, it is unclear to what degree the task involves allocentric spatial memory and/or egocentric spatial memory. More specifically, a rat may remember what bar it just pressed on the basis of extramaze cues in the environment (allocentric), or a rat may remember on the basis of cues related to a particular body movement (egocentric). In accord with the findings in the radial arm maze, Broersen et al. (1995) demonstrated that scopolamine infusions into the prelimbic area pro- 
duced a memory deficit in the matching-to-position task in a dose- and delay-dependent manner. In contrast, a recent study found that scopolamine injections into the prelimbic area did not impair delayed nonmatching-toposition performance (Aura \& Reikkinen, 1999). However, in this experiment, only one dose of scopolamine was injected, which raises the possibility that the higher doses of scopolamine used in other studies that used a nonmatching-to-sample procedure and found memory deficits (Brito et al., 1989; Broersen et al., 1995; Granon et al., 1995) might have produced a working memory impairment.

The findings from experiments employing intracranial drug infusions on working memory tasks provide evidence that muscarinic cholinergic transmission in the prelimbic and infralimbic areas is important for working memory (Granon et al., 1995; Ragozzino \& Kesner, 1998). This is further supported by results indicating that blockade of muscarinic receptors in the prelimbic area impairs working memory but does not impair visuospatial discriminations in a T-maze or a radial arm maze (Brito et al., 1989; Granon et al., 1995; Ragozzino \& Kesner, 1998). For example, scopolamine infusions into the prelimbicinfralimbic areas do not impair performance when a rat must remember always to enter one spatial location for a reinforcement and to avoid entering another spatial location. Furthermore, acetylcholine release in the prelimbic area does not change from basal levels when rats perform a visual discrimination task (Himmelheber, Sarter, $\&$ Bruno, 1997). These findings suggest that cholinergic transmission - and, in particular, muscarinic cholinergic transmission-in the prelimbic-infralimbic areas is not critical for retention of a discrimination, as well as perceptual, motivational, or motor functions, but is important for spatial working memory.

Compared with muscarinic receptors, few studies have examined the role that nicotinic cholinergic receptors in the prelimbic-infralimbic areas play in working memory. One experiment in which the effects of nicotinic cholinergic transmission in the prelimbic area on working memory were investigated found that blockade of nicotinic receptors impairs spatial working memory in a delayed matching-to-sample procedure, but not in a delayed nonmatching-to-sample procedure (Granon et al., 1995). The reason for this selective deficit is unclear. Poucet and colleagues (Granon et al., 1995) have proposed that because a matching-to-sample rule requires a rat to go against its tendency to alternate, this procedure may be more difficult in that it makes greater attentional demands and/or requires a rat to shift strategies. Manipulations of the prelimbic area have been found to impair performance on tasks that either accentuate attentional processes or require a shift in strategies (de Bruin, Sánchez-Santed, Heinsbroek, Donker, \& Postmes, 1994; Muir et al., 1996; Ragozzino, Detrick, \& Kesner, 1999a; Ragozzino, Wilcox, et al., 1999). On the basis of the performance of control rats, there is a trend for the matching-to-sample procedure to be more difficult than the nonmatching-to-sample procedure (Granon et al., 1995). However, future experiments are needed in which changes in attentional demands and strategy selection, as well as their interactions, are systematically examined to better determine what role nicotinic cholinergic receptors in the prelimbic area play in cognitive processes.

\section{Cholinergic Influences in the Prelimbic and Infralimbic Areas on Attention}

Damage to basal forebrain cholinergic neurons impairs performance on different attention tasks, and because these neurons project to the medial prefrontal cortex, it has been assumed that the medial prefrontal cortex is also involved in some aspect of attention (Chiba, Bucci, Holland, \& Gallagher, 1995; McGaughy, Kaiser, \& Sarter, 1996; Muir, Everitt, \& Robbins, 1994; Muir, Page, Sirinathsinghji, Robbins, \& Everitt, 1993; Robbins et al., 1989; Stoehr et al., 1997; Turchi \& Sarter, 1997). Indeed, medial prefrontal cortex lesions, centered in the prelimbic area, have been found to impair attention (Muir et al., 1996). The deficits following lesions centered in the prelimbic area were observed on a five-choice serial reaction time task that required sustained attention to a brief light stimulus across five different spatial locations. These lesions impaired choice accuracy and increased perseverative responding. Furthermore, in the lesioned rats, correct response latency increased, and there was a strong trend for accuracy to decline when attentional demands were augmented.

A series of recent experiments by Givens and colleagues (Gill, Masters, Sarter, \& Givens, 1999; Gill, Sarter, \& Givens, in press; Williams, Mohler, \& Givens, 1999) examined the role of cholinergic input to different medial prefrontal cortex subregions in attentional processes. In one study, rats were trained to press one of three bars when a brief light stimulus appeared above it (Williams et al., 1999). The light stimulus occurred for a 200- or 500-msec duration and was presented in either a block of predictable trials, where the light stimulus was presented for the same duration for all the trials, or a block of random trials, where the light stimulus was presented for either 200 or $500 \mathrm{msec}$ in a random fashion. Scopolamine infusions into the prelimbic area reduced choice accuracy in the random condition, but not in the predictable condition, suggesting that muscarinic cholinergic transmission in the prelimbic area is important under conditions in which there are increased attentional demands (Williams et al., 1999). These results are comparable with the lesion study discussed earlier, which demonstrated that lesions centered in the prelimbic area impaired response selection with enhanced attentional demands (Muir et al., 1996).

In another study by this group, recordings of prelimbic single-unit activity during sustained attention performance before and after unilateral cholinergic deafferentation of the medial prefrontal cortex were examined (Gill et al., in press). In this experiment, rats were tested on a visual stimulus detection task. If a visual stimulus $(25$, 50 , or $500 \mathrm{msec}$ in duration) was presented on a trial, a rat had to press one of two bars in an operant chamber to re- 
ceive a water reinforcement. If no visual stimulus was presented on a trial, a rat had to press the other bar. During certain trials, an overhead flashing light was presented as a visual distractor to increase attentional demands. Several neurons in the prelimbic region exhibited correlated activity either when the rats were preparing to make a response or during response emission, consistent with the idea that the prefrontal cortex is involved in the planning and execution of goal-directed behavior. In addition, an even greater number of prelimbic neurons showed correlated activity in anticipation of a reward and during consumption of a reward (Gill et al., in press). In contrast, unit activity did not correlate with the presentation of sensory stimuli. Nevertheless, presentation of the visual distractor did lead to a decrement in performance and a simultaneous altering of the firing rate of several prefrontal cells.

Injecting the cholinergic neurotoxin 192 IgG-saporin into the medial prefrontal cortex produced a significant reduction in the response- and reward-correlated activity of these prelimbic neurons. Cholinergic deafferentation also decreased the proportion of neurons modulated by the presentation of the distractor. Moreover, those neurons that did change their activity in the presence of the distractor showed a reduced magnitude of change, as compared with those recorded prior to the cholinergic lesion. However, although a unilateral cholinergic lesion of the medial prefrontal cortex significantly altered the response properties of prelimbic neurons during a sustained attention task, this lesion did not impair task performance. More recently, preliminary results from a subsequent study indicate that bilateral infusions of 192 IgG-saporin into the medial prefrontal cortex do impair sustained attention (Gill et al., 1999). Taken together, these results suggest that neuronal activity in the prelimbic area is related to sustained attention and that cholinergic modulation of cellular firing in this subregion may be important for accurate performance on tasks requiring sustained attention (Gill et al., 1999; Gill et al., in press). Important to note, however, because the neurotoxin was infused into multiple subregions within the medial prefrontal cortex, areas outside the prelimbic area may also have contributed to the impairing effects of cholinergic deafferentation on attentional processing.

Nonetheless, experiments measuring acetylcholine output by in vivo microdialysis with high pressure liquid chromatography also implicate prelimbic cholinergic afferents in attention. In particular, a study in which acetylcholine output was assessed in the prelimbic area during a sustained attention task found that acetylcholine output increased when the attentional demands were increased by adding a flashing light during the task (Sarter et al., 1996). This result contrasts with findings discussed earlier that acetylcholine output in the prelimbic area did not change when a flashing light was presented during a "simple" discrimination task (Himmelheber et al., 1997). In the sustained attention task, a rat had to differentially respond to receive a reinforcement, depending on the type of signal presented. However, in the discrimination task, the rule was to execute the same response when a signal appeared. Thus, one possibility is that the differential response selection task required greater attentional processing and, concomitantly, higher levels of acetylcholine in the prelimbic area (Sarter et al., 1996).

Changes in acetylcholine output were also observed in other conditions not directly related to task performance (Sarter et al., 1996). Acetylcholine output was enhanced when rats were moved into the test chamber and at the initial onset of a test session (Sarter et al., 1996). This increase in acetylcholine release may be related to attention or arousal mechanisms activated when animals are moved to a different environment.

\section{Cholinergic Influences in the \\ Anterior Cingulate on Working Memory}

In one recent experiment, the effects of cholinergic drug infusions into the anterior cingulate on working memory were assessed in a spatial memory task using a 12-arm radial maze. In contrast to infusions into the prelimbic areas, injections of scopolamine into the dorsal anterior cingulate did not impair working memory in a spatial continuous recognition test (Ragozzino \& Kesner, 1998). In another experiment, the effects of muscarinic cholinergic receptor blockade in the dorsal anterior cingulate during a delayed matching-to-position task was determined. In this task, rats were tested in an operant chamber that had two retractable levers. During the sample phase of a trial, one of the two levers was presented. After a response on the sample lever, the level was retracted. Following a delay, both levers were presented during the test phase. To receive a reinforcement, a rat had to press the same lever as that in the sample phase. Scopolamine injections into the dorsal anterior cingulate impaired performance on a delayed matching-to-position task, but the deficit was delay independent, suggesting that the muscarinic receptors in this area were not specifically critical for working memory performance (Herremans, Hijzen, \& Olivier, 1997; Herremans, Welborn, Hijzen, Olivier, \& Slangen, 1996). Similarly, Pepeu and colleagues (1996) measured acetylcholine output, using a transverse dialysis probe centered in the dorsal and ventral anterior cingulate. It was demonstrated that acetylcholine release was increased during spontaneous alternation performance in a Y-maze, a task that has a spatial working memory component (Ragozzino, Unick, \& Gold, 1996). However, it is unclear whether the change in acetylcholine output was related to working memory, novelty, or some combination. Acetylcholine levels were measured in this study during the rat's first exposure to the maze, and an increase in acetylcholine release is commonly observed in several forebrain regions when rats are placed in a novel or different environment (Acquas, Wilson, \& Fibiger, 1996; Aloisi, Casamenti, Scali, Pepeu, \& Carli, 1997; Orsetti, Casamenti, \& Pepeu, 1996; Sarter et al., 1996).

When evaluated in conjunction with other studies indicating that anterior cingulate lesions do not impair spatial working memory (Ragozzino et al., 1998; SánchezSanted, de Bruin, Heinsbroek, \& Verwer, 1997; Silva 
et al., 1986), these results suggest that cholinergic transmission in the anterior cingulate does not modulate spatial working memory processes, as was observed in the prelimbic and infralimbic areas (Herremans et al., 1997; Herremans et al., 1996; Ragozzino \& Kesner, 1998). Rather, the findings raise the possibility that cholinergic input to the medial prefrontal cortex modulates different cognitive processes determined by the particular prefrontal cortex subregion. Thus, different prefrontal cortex subregions may differentially contribute to various cognitive functions that can be modulated by cholinergic input to those subregions.

\section{Cholinergic Influences in the Anterior Cingulate on Attention}

The behavioral effects of muscarinic cholinergic blockade in the anterior cingulate on different discrimination tasks appear to be more related to changes in attention, as opposed to working memory (Herremans et al., 1997; Herremans et al., 1996). More specifically, Herremans and colleagues found that scopolamine infusions into the anterior cingulate during a matching-to-position or a tone-light conditional discrimination task decreased discriminibility at no delay and appeared to produce a general increase in distractability. In another experiment in which the effects of scopolamine infusions into the anterior cingulate on attention were investigated, it was found that muscarinic cholinergic blockade in this subregion impaired performance during a varying stimulus duration condition, but not during a predictable stimulus duration condition (Williams et al., 1999). Like the findings in the prelimbic area, these results suggest that muscarinic cholinergic transmission in the anterior cingulate is important when there is an increase in attentional demands (Williams et al., 1999).

Further support for the idea that cholinergic input to the anterior cingulate modulates attentional processes comes from an experiment by Pirch and colleagues (Pirch, Turco, \& Rucker, 1992) in which single neurons in the anterior cingulate were recorded during an auditory discrimination task. Neurons in the anterior cingulate were found to exhibit discrimination-related responses to conditioned tones, which were modulated by direct application of acetylcholine. These acetylcholine-induced changes were blocked by the muscarinic antagonist atropine (Pirch et al., 1992).

In other experiments, acetylcholine output increased in the anterior cingulate during exposure to novel sensory stimuli or environments (Acquas et al., 1996; Giovannini, Bartolini, Kopf, \& Pepeu, 1998; Inglis \& Fibiger, 1995). Conversely, there was no change in acetylcholine output after extensive exposure to unconditioned stimuli (Acquas et al., 1996). Novel conditions are commonly interpreted as highly arousing and may engage attentional processes for exploring particular environments or conditions. Thus, the changes in acetylcholine output in the anterior cingulate during novel situations may reflect an increase in attentional demands. Whether the novelty-induced changes in acetylcholine release in different medial prefrontal subregions are related to the same processes (i.e., attention) or to different processes is still to be determined.

\section{THE ROLE OF DOPAMINERGIC TRANSMISSION IN THE PREFRONTAL CORTEX ON COGNITIVE PROCESSES}

A few behavioral experiments have studied the influence of dopamine transmission in the medial prefrontal cortex on working memory and learning. In addition to traditional pharmacological approaches, in vivo microdialysis, in vivo voltammetry, and the use of the catecholamine neurotoxin 6-OHDA have provided approaches for understanding how dopaminergic transmission in separate prefrontal cortex subregions influences different behavioral and cognitive processes.

\section{Dopaminergic Influences in the Prelimbic and Infralimbic Areas on Working Memory}

Current findings suggest that dopamine afferents in the prelimbic-infralimbic areas modulate working memory. One of the first experiments to suggest a role of prelimbic-infralimbic dopamine in working memory demonstrated that dopamine depletion in the prelimbicinfralimbic areas impaired delayed alternation in both a T-maze and a radial arm maze (Busber \& Schmidt, 1990). In this experiment, the lesion-induced deficit was selective to working memory, since these same lesions did not affect performance on alternation tests without delays. In another study, Sokolowski and Salamone (1994) demonstrated that dopamine depletion, by use of 6-OHDA, in the prelimbic-infralimbic areas impaired performance on a differential reinforcement of low rates of responding task with a 30 -sec schedule. In this task, a rat must withhold responding until $30 \mathrm{sec}$ elapses to receive a reinforcement. This test involves memory for temporal duration and likely recruits working memory processes. Although this task has also been used to measure impulsivity, it is important to note in this study that lesioned rats were more likely to respond before $30 \mathrm{sec}$ had elapsed but did not exhibit any motor abnormalities (i.e., hyperactivity), suggesting that this deficit was selective to temporal discrimination (Sokolowski \& Salamone, 1994).

The results from investigations using intracranial drug injections suggest that $D_{1}$ receptors in these areas are particularly critical for working memory performance. In examining working memory with a delayed matchingto-position procedure, infusions of SCH-23390, a dopamine $D_{1}$ antagonist, into the prelimbic area impaired performance (Broersen et al., 1995). The drug-induced deficit was dose dependent, and there was a trend for the impairment to be delay dependent (Broersen et al., 1995). Blockade of $D_{1}$ receptors in the prelimbic area has also been found to produce spatial working memory deficits in a dose-dependent manner (Seamans, Floresco, \& Phillips, 1998). Infusions of a full $D_{1}$ receptor agonist into the prelimbic area likewise produced spatial working memory impairments (Zahrt, Taylor, Mathew, \& Arnsten, 1997). These results suggest that there is an optimal window of 
dopamine $D_{1}$ receptor stimulation in the prelimbic area for facilitation of working memory and that either a paucity or an excess of prelimbic $D_{1}$ receptor stimulation may impair performance.

In contrast to $D_{1}$ receptors, infusions of the $D_{2}$ antagonist sulpiride into the prelimbic area do not impair working memory (Brito et al., 1989; Seamans et al., 1998). Unknown is what effect selective $\mathrm{D}_{3}, \mathrm{D}_{4}$, and $\mathrm{D}_{5}$ agents infused into the prelimbic-infralimbic areas would have on working memory. Thus, to date it appears that dopamine stimulation of prelimbic $D_{1}$ receptors plays a role in working memory and that there is a limited range of $D_{1}$ receptor stimulation that is optimal for working memory performance.

\section{Dopaminergic Influences in the \\ Prelimbic-Infralimbic Areas on Learning}

A recent experiment examined the effects of 6-OHDA lesions centered in the prelimbic-infralimbic areas on acquisition and expression of conditioned fear, using tone-shock pairings (Morrow, Elsworth, Rasmusson, \& Roth, 1999). Dopamine depletion did not impair the learning or expression of a conditioned fear response. However, dopamine depletion did impair the extinction of fear conditioning. These findings suggest that dopamine input to the prelimbic-infralimbic areas may be critical for behavioral flexibility - that is, adapting behavior to changes in environmental conditions, rather than the learning of conditioned fear. Further support for this idea comes from a preliminary study in which the effects of $D_{1}$ receptor blockade in the learning and reversal of behavioral rules were examined (Ragozzino, Detrick, \& Kesner, 1999b). Blockade of $D_{1}$ receptors in the prelimbic-infralimbic areas did not impair learning of a visual cue or response (turn) discrimination in a cross-maze but did impair performance when rats had to shift from one discrimination strategy to the other discrimination strategy (Ragozzino, Detrick, \& Kesner, 1999b).

In contrast to the experiments described above, the findings from in vivo microdialysis studies during acquisition suggest a role for prelimbic-infralimbic dopamine release in learning. In assessing acquisition of conditioned fear, dopamine was observed to increase during the presentation of a stimulus (tone or flashing light) not paired with footshock, with further increases in dopamine release when the stimulus was paired with footshock (Wilkinson et al., 1998). This change in dopamine output diminished across conditioning trials in conjunction with enhanced freezing, which was used as a measure of conditioned fear. These findings suggest that dopamine release in the prelimbic-infralimbic areas contributes to new learning.

Dopamine output was also found to increase during acquisition of a barpress operant under a continuous reinforcement schedule (Izaki, Hori, \& Nomura, 1998). The observed increase in dopamine release across learning contrasts with the experiment by Wilkinson et al. (1998), which found the opposite pattern during conditioned fear acquisition. Because the tasks had different types of re- inforcement (aversive vs. appetitive), one possibility is that the dynamics of dopamine release for learning an aversive task is different from that in an appetitively motivated task. During a retention session in the operant task, there was no change in dopamine output from basal levels (Izaki et al., 1998). These results support the idea that dopamine output in the prelimbic-infralimbic areas is related to stimulus-reward learning, but not for retrieval of the learned association.

Although the results from studies suggesting that prelimbic-infralimbic dopamine release is involved in learning appear to conflict with experiments demonstrating that 6-OHDA lesions or dopamine receptor blockade do not impair acquisition, the two sets of findings may actually be related. More specifically, in the conditioned fear study in which dopamine output was concomitantly measured with freezing behavior, rats were exposed to the test apparatus for several minutes without any exposure to shock (Wilkinson et al., 1998). Subsequently, rats were presented a stimulus with footshock. The increase in dopamine release with the first presentation of the stimulusshock pairing may be related to the elicitation of a new response pattern induced by changes in the environmental conditions. Similarly, in the operant task, rats were exposed to the operant chamber on several sessions, receiving a reinforcement under a variable interval schedule without barpress training (Izaki et al., 1998). During the acquisition session, rats now had to press a bar to receive a reinforcement under a continuous reinforcement schedule. Thus, in this experiment, the conditions were also changed, requiring a different response pattern. Taken together, the findings suggest that changes in dopamine release in the prelimbic-infralimbic areas may underlie the flexible shifting of a learning set to changes in environmental demands.

The idea that dopamine output plays a role in behavioral flexibility is further supported by a recent study using in vivo voltammetry (Richardson \& Gratton, 1998). In vivo voltammetry has advantages over in vivo microdialysis in that dopamine changes can be observed in the millisecond range versus the minute range, and the spatial resolution is also better. In this study, rats were trained to barpress for a liquid reinforcement under different reinforcement schedules. Changes in dopamine release in the prelimbic or the infralimbic area occurred when the temporal component of the learned association was changed or the reinforcement that a rat expected was changed. Dopamine release did not change during presentation of the conditioned stimuli or consumption of the liquid reinforcement. Thus, dopamine release selectively changed when the learning conditions were modified (Richardson \& Gratton, 1998). These findings provide additional evidence that dynamic changes in prelimbic dopamine release may underlie behavioral flexibility.

\section{Dopaminergic Influences in the Anterior Cingulate on Working Memory}

In one experiment, the effects of SCH-23390 infusions into the anterior cingulate on a delayed matching- 
to-position task in an operant chamber were examined. The procedure was the same as that described earlier. Blockade of $D_{1}$ receptors did not impair working memory performance (Herremans et al., 1996). Importantly, the drug doses used in this experiment were doses previously found to affect behavioral performance when injected into other brain sites, suggesting that activation of dopaminergic receptors in the anterior cingulate does not influence working memory. Moreover, these findings are consistent with lesion studies demonstrating that anterior cingulate lesions do not impair working memory (Ragozzino et al., 1998; Sánchez-Santed et al., 1997; Silva et al., 1986).

\section{SUMMARY AND CONCLUSIONS}

In recent years, several experiments have attempted to unravel how cholinergic and dopaminergic afferents in different subregions of the rat prefrontal cortex contribute to learning, memory, and attention. Activation of cholinergic or dopaminergic receptors in the prelimbic and infralimbic areas facilitates working memory. In particular, numerous findings suggest that activation of muscarinic cholinergic or activation of $D_{1}$ dopamine receptors in the prelimbic-infralimbic areas contributes to working memory. In contrast, stimulation of $\mathrm{D}_{2}$ receptors in the prelimbic-infralimbic areas does not appear to play a significant role in working memory. Less is known about the contribution of nicotinic cholinergic receptors to working memory.

More extensive research is needed to determine whether acetylcholine and dopamine directly interact within the prefrontal cortex to facilitate working memory or whether their actions are independent. Some experiments suggest an interaction between acetylcholine and dopamine in the medial prefrontal cortex, whereas other studies do not (Moore, Fadel, Sarter, \& Bruno, 1999; Yang \& Mogenson, 1990).

Cholinergic input to the prelimbic-infralimbic areas may also influence attentional processing, particularly when there is an increase in attentional demands. However, it is possible that cholinergic input more generally influences behavioral flexibility, only incidently affecting attentional processes. This is based on previous findings indicating that medial prefrontal lesions, centered in the prelimbic-infralimbic areas, produced random barpressing, an effect not influenced by manipulations that increase attentional demands (Miner, Ostrander, \& Sarter, 1997).

Activation of dopamine receptors in the prelimbicinfralimbic areas may also contribute to behavioral flexibility. Different lines of evidence suggest that there are dynamic changes in dopamine release in the prelimbicinfralimbic areas when there are changes in environmental demands. In addition, preliminary evidence suggests that activation of $D_{1}$ receptors may facilitate behavioral flexibility or strategy switching.

In contrast to the prelimbic and infralimbic areas, the cholinergic and dopaminergic input to the anterior cingu- late does not appear to play a critical role in spatial working memory. Rather, cholinergic input to the anterior cingulate seems intimately involved in attentional processing. More specifically, activation of muscarinic cholinergic receptors occurs when there is an increase in attentional demands. These results also suggest that whatever attentional requirements there are in most working memory tests, they are different from the attentional processes mediated by the anterior cingulate. Examining the role of nicotinic and dopamine receptors will be important to building a more complete picture of cholinergic and dopaminergic modulation of anterior cingulate activity related to attention.

Overall, the findings suggest that acetylcholine and dopamine modulate activity in the medial prefrontal cortex to facilitate different behavioral and cognitive processes. Importantly, current findings, particularly in the prelimbic and infralimbic regions, suggest that the same prefrontal cortex subregions may contribute to various functionsthat is, working memory and strategy selection-under different dynamic states and that cholinergic and dopaminergic afferents influence these different states of activity. However, there is still the possibility that the diverse behavioral deficits produced by manipulations of the prelimbic-infralimbic areas represent disruption of a single process (i.e., attention). Future experiments will be important in further elucidating the role of prefrontal cholinergic and dopaminergic afferents in modulating cognitive processes.

\section{REFERENCES}

Acquas, E., Wilson, C., \& Fibiger, H. C. (1996). Conditioned and unconditioned stimuli increase frontal cortical and hippocampal acetylcholine release: Effects of novelty, habituation and fear. Journal of Neuroscience, 16, 3088-3096.

Aloisi, A. M., Casamenti, F., Scali, C., Pepeu, G., \& Carli, G. (1997). Effects of novelty, pain and stress on hippocampal extracellular acetylcholine levels in male rats. Brain Research, 748, 219-226.

AURA, J., \& RIEKKINEN, P. (1999). Blockade of NMDA receptors located at the dorsomedial prefrontal cortex impairs spatial working memory in rats. NeuroReport, 10, 243-248.

Berger, B., Gaspar, P., \& Verney, C. (1991). Dopaminergic innervation of the cerebral cortex: Unexpected differences between rodents and primates. Trends in Neurosciences, 14, 2-27.

Brito, G. N. O., \&, Brito, L. S. O. (1990). Septohippocampal and the prelimbic sector of frontal cortex: A neuropsychological battery analysis in the rat. Behavioural Brain Research, 36, 127-146.

Brito, G. N. O., Silva, S. P., \& Brito, L. S. (1989). The cholinergic and dopaminergic systems of the prelimbic sector of frontal cortex and memory in the rat. Brazilian Journal of Medical Biological Research, 22, $1155-1158$.

Broersen, L. M., Heinsbroek, R. P. W., De Bruin, J. P. C., Uylings, H. B. M., \& OLIVIER, B. (1995). The role of the medial prefrontal cortex of rats in short-term memory functioning: Further support for involvement of cholinergic, rather than dopaminergic mechanisms. Brain Research, 674, 221-229.

Burgess, P. W., \& Shallice, T. (1996). Response suppression, initiation and strategy use following frontal lobe lesions. Neuropsychologia, 34, 263-273.

Busber, M., \& SCHMidt, W. J. (1990). 6-OHDA lesions of the rat prefrontal cortex increases locomotor activity, impairs acquisition of delayed alternation tasks, but does not affect uninterrupted tasks in the radial maze. Behavioural Brain Research, 37, 157-168. 
Bussey, T. J., Muir, J. L., Everitt, B. J., \& Robbins, T. W. (1997) Triple dissociation of anterior cingulate, posterior cingulate, and medial prefrontal cortices on visual discrimination tasks using a touchscreen testing procedure for the rat. Behavioral Neuroscience, 111, 920-936.

ChAO, L. L., \& KNiGHT, R. T. (1995). Human prefrontal lesions increase distractibility to irrelevant sensory inputs. NeuroReport, 6, 16051610.

Chiba, A. A., Bucci, D. J., Holland, P. C., \& Gallagher, M. (1995). Basal forebrain cholinergic lesions disrupt increments but not decrements in conditioned stimulus processing. Journal of Neuroscience, 15, 7315-7322.

Damasio, A. R., Tranel, D., \& Damasio, H. (1990). Individuals with sociopathic behavior caused by frontal damage fail to respond autonomically to social stimuli. Behavioural Brain Research, 41, 81-94.

de Bruin, J. P. C., Sánchez-Santed, F., Heinsbroek, R. P. W., DONKER, A., \& Postmes, P. (1994). A behavioural analysis of rats with damage to the medial prefrontal cortex using the Morris water maze: Evidence for behavioural flexibility, but not for impaired spatial navigation. Brain Research, 652, 323-333.

DE Bruin, J. P. C., SWinkels, W. A. M., \& DeBrabander, J. M. (1997). Response learning of rats in a Morris water maze: Involvement of the medial prefrontal cortex. Behavioural Brain Research, 85, 47-55.

DeCoteau, W. E., Kesner, R. P., \& Williams, J. M. (1997). Shortterm memory for food reward magnitude: The role of the prefrontal cortex. Behavioural Brain Research, 88, 239-249.

Delatour, B., \& Gisquet-Verrier, P. (1996). Prelimbic cortex specific lesions disrupt delayed-variable response tasks in the rat. Behavioral Neuroscience, 110, 1282-1298.

DEMBER, W. N., \& FowLER, H. (1958). Spontaneous alternation behavior. Psychological Bulletin, 55, 412-428.

Dias, R., Robbins, T. W., \& RobERTS, A. C. (1996). Dissociation in prefrontal cortex of affective and attentional shifts. Nature, 380, 69-72.

Eichenbaum, H., ClegG, R. A., \& Feeley, A. (1983). Reexamination of functional subdivisions of the rodent prefrontal cortex. Experimental Neurology, 79, 434-451.

Freedman, M., Black, S., Ebert, P., \& Binns, M. (1998). Orbitofrontal function, object alternation and perseveration. Cerebral Cortex, 8, 18-27.

FryszTaK, R. J., \& NEAFsey, E. J. (1994). The effect of medial frontal cortex lesions on cardiovascular conditioned emotional responses in the rat. Brain Research, 643, 181-193.

FUSTER, J. M. (1997). The prefrontal cortex (3rd Ed.). New York: Raven.

Gallagher, M., McMahan, R. W., \& Schoenbaum, G. (1999). Orbitofrontal cortex and representation of incentive value in associative learning. Journal of Neuroscience, 19, 6610-6614.

Gaykema, R. P. A., Luiten, P. G. M., Nyakas, C., \& Traber, J. (1990). Cortical projection patterns of the medial septum-diagonal band complex. Journal of Comparative Neurology, 293, 103-124.

Gill, T. M., Masters, J. J., SARTER, M., \& Givens, B. (1999). The role of acetylcholine within the medial prefrontal and posterior parietal cortices during sustained visual attention in the rat. Society for Neuroscience Abstracts, 25, 1895.

Gill, T. M., SARTer, M., \& Givens, B. (in press). Sustained visual attention performance-associated prefrontal neuronal activity: Evidence for cholinergic modulation. Journal of Neuroscience.

Giovannini, M. G., Bartolini, L., KoPf, S. R., \& PePeu, G. (1998). Acetylcholine release from the frontal cortex during exploratory activity. Brain Research, 784, 218-227.

Granon, S., \& Poucet, B. (1995). Medial prefrontal lesions in the rat and spatial navigation: Evidence for impaired planning. Behavioral Neuroscience, 108, 478-484.

Granon, S., Poucet, B., Thinus-Blanc, C., Changeux, J. P., \& VIDAL, C. (1995). Nicotinic and muscarinic receptors in the rat prefrontal cortex: Differential roles in working memory, response selection and effortful processing. Psychopharmacology, 119, 139-144.

GroENEWEGEN, H. J. (1988). Organization of the afferent connections of the mediodorsal thalamic nucleus in the rat, related to the mediodorsalprefrontal topography. Neuroscience, 24, 379-431.

Herremans, A. H. J., Hijzen, T. H., \& Olivier, B. (1997). Effects of cholinergic drug infusions into the dorsal part of the medial prefrontal cortex on delayed conditional discrimination performance in the rat. Behavioural Brain Research, 84, 291-299.

Herremans, A. H. J., Welborn, P. F. E., Hijzen, T. H., Olivier, B., \& SLANGEN, J. L. (1996). Effects of infusion of cholinergic drugs into the prefrontal cortex area on delayed matching to position performance in the rat. Brain Research, 711, 102-111.

Himmelheber, A. M., SARTer, M., \& Bruno, J. P. (1997). Operant performance and cortical acetylcholine release: Role of response rate, reward density and non-contingent stimuli. Cognitive Brain Research, 6, 23-36.

INGLIS, F. M., \& Fibiger, H. C. (1995). Increases in hippocampal and frontal cortical acetylcholine release associated with presentation of sensory stimuli. Neuroscience, 66, 81-86.

IZAKI, Y., Hori, K., \& Nomura, M. (1998). Dopamine and acetylcholine elevation on lever-press acquisition in rat prefrontal cortex. Neuroscience Letters, 258, 33-36.

KesNer, R. P., \& Holbrook, T. (1987). Dissociation of item and order spatial memory in rats following medial prefrontal cortex lesions. Neuropsychologia, 25, 653-664.

Kesner, R. P., Hopkins, R. O., \& Fineman, B. (1994). Item and order dissociation in humans with prefrontal cortex damage. Neuropsychologia, 32, 881-891.

Kesner, R. P., Hunt, M. E., Williams, J. M., \& Long, J. M. (1996) Prefrontal cortex and working memory for spatial response, spatial location, and visual object information in the rat. Cerebral Cortex, 6 , 311-318.

KolB, B. (1984). Functions of the frontal cortex of the rat: A comparative review. Brain Research Reviews, 8, 65-98.

KolB, B. (1990). Animal models for human PFC-related disorders. Progress in Brain Research, 85, 501-519.

Kolb, B., Buhrmann, K., McDonald, R., \& Sutherland, R. J. (1994). Dissociation of the medial prefrontal, posterior parietal, and posterior temporal cortex for spatial navigation and recognition memory in the rat. Cerebral Cortex, 6, 664-680.

Krettek, J. E., \& Price, J. L. (1977). The cortical projections of the mediodorsal nucleus and adjacent thalamic nuclei in the rat. Journal of Comparative Neurology, 171, 157-192.

LEONARD, C. M. (1969). The prefrontal cortex of the rat: I. Cortical projection of the mediodorsal nucleus: II. Efferent connections. Brain Research, 12, 321-343.

McDowell, S., Whyte, J., \& D'Esposito, M. (1998). Differential effect of a dopaminergic agonist on prefrontal function in traumatic brain injury patients. Brain, 121, 1155-1164.

MCGAughy, J., KAISER, T., \& SARTER, M. (1996). Behavioral vigilance following infusions of $192 \mathrm{IgG}$-saporin into the basal forebrain: Selectivity of the behavioral impairment and relation to cortical AchEpositive fiber density. Behavioral Neuroscience, 110, 247-265.

Milner, B., Petrides, M., \& Smith, M. L. (1985). Frontal lobes and temporal organization of memory. Human Neurobiology, 4, 137-142.

Miner, L. A. H., Ostrander, M., \& SarTer, M. (1997). Effects of ibotenic acid-induced loss of neurons in the medial prefrontal cortex of rats on behavioural vigilance: Evidence for executive dysfunction. Journal of Psychopharmacology, 11, 169-178.

Mogenson, J., \& Holm, S. (1994). The prefrontal cortex and variants of sequential behaviour: Indications of functional differentiation between subdivisions of the rat's prefrontal cortex. Behavioural Brain Research, 63, 89-100.

Moore, H., Fadel, J., Sarter, M., \& Bruno, J. P. (1999). Role of accumbens and cortical dopamine receptors in the regulation of cortical acetylcholine release. Neuroscience, 88, 81 1-822.

Morrow, B. A., Elsworth, J. D., Rasmusson, A. M., \& Roth, R. H. (1999). The role of mesoprefrontal dopamine neurons in the acquisition and expression of conditioned fear in the rat. Neuroscience, 92 , 553-564.

Muir, J. L., Everitt, B. J., \& RobBins, T. W. (1994). AMPA-induced excitotoxic lesions of the basal forebrain: A significant role for the cortical cholinergic system in attention function. Journal of Neuroscience, 14, 2313-2326.

Muir, J. L., EveritT, B. J., \& RobBins, T. W. (1996). The cerebral cortex of the rat and visual attentional function: Dissociable effects of mediofrontal, cingulate, anterior dorsolateral, and parietal cortex le- 
sions on a 5-choice serial reaction time task. Cerebral Cortex, 6,470 481 .

Muir, J. L., Page, K. J., Sirinathsinghi, D. J. S., Robbins, T. W., \& EVERITT, B. J. (1993). Excitotoxic lesions of basal forebrain cholinergic neurons: Effects on learning, memory and attention. Behavioural Brain Research, 57, 123-131.

Olton, D. S., \& Schlosberg, P. (1978). Food searching strategies in young rats: Win-shift performance predominates over win-stay. Journal of Comparative \& Physiological Psychology, 92, 609-618.

Orsetti, M., Casamenti, F., \& PePeu, G. (1996). Enhanced acetylcholine release in the hippocampus and cortex during acquisition of an operant behavior. Brain Research, 724, 89-96.

OWEN, A. M. (1997). Cognitive planning in humans: Neuropsychological, neuroanatomical and neuropharmacological perspectives. Progress in Neurobiology, 53, 431-450.

Owen, A. M., Morris, R. G., Sahakian, B. J., Polker, C. E., \& RobBINS, T. W. (1996). Double dissociations of memory and executive functions in working memory tasks following frontal lobe excisions, temporal lobe excisions or amygdalo-hippocampectomy in man. Brain, 119, 1597-1615.

Petrides, M., Alivisatos, B., Evans, A. C., \& Meyer, E. (1993). Dissociation of human mid-dorsolateral from posterior dorsolateral frontal cortex in memory processing. Proceedings of the National Academy of Sciences, 90, 873-877.

Pirch, J. H., Turco, K., \& Rucker, H. K. (1992). A role for acetylcholine in conditioning-related responses of rat frontal cortex neurons: Microiontophoretic evidence. Brain Research, 586, 19-26.

Preuss, T. M. (1995). Do rats have prefrontal cortex? The RoseWoosley-Akert program reconsidered. Journal of Cognitive Neuroscience, 7, 1-24.

Ragozzino, M. E., Adams, S., \& Kesner, R. P. (1998). Differential involvement of the dorsal anterior cingulate and prelimbic-infralimbic areas of the rodent prefrontal cortex in spatial working memory. $\mathrm{Be}$ havioral Neuroscience, 112, 293-303.

Ragozzino, M. E., Detrick, S., \& Kesner, R. P. (1999a). Involvement of the prelimbic-infralimbic areas of the rodent prefrontal cortex in behavioral flexibility for place and response learning. Journal of Neuroscience, 19, 4585-4594.

Ragozzino, M. E., Detrick, S., \& Kesner, R. P. (1999b). The role of prelimbic-infralimbic dopamine $\mathrm{D}_{1}$ receptors in behavioral flexibility. Society for Neuroscience Abstracts, 25, 890.

RaGozzino, M. E., \& KeSNer, R. P. (1998). The effects of muscarinic cholinergic receptor blockade in the rat anterior cingulate and prelimbic/infralimbic cortices on spatial working memory. Neurobiology of Learning \& Memory, 69, 241-257.

Ragozzino, M. E., Unick, K. E., \& Gold, P. E. (1996). Hippocampal acetylcholine release during memory testing in rats: Augmentation by glucose. Proceedings of the National Academy of Sciences, 93, 4693-4698

Ragozzino, M. E., Wilcox, C., Raso, M., \& Kesner, R. P. (1999). Involvement of rodent prefrontal cortex subregions in strategy switching. Behavioral Neuroscience, 113, 32-41.

Richardson, N. R., \& Gratton, A. (1998). Changes in medial prefrontal cortical dopamine levels associated with response-contingent food reward: An electrochemical study in rat. Journal of Neuroscience, 18, 9130-9138.

Robbins, T. W., Everitt, B. J., Marston, H. M., Wilkinson, J., Jones, G. H., \& PAGE, K. J. (1989). Comparative effects of ibotenic acidand quisqualic acid-induced lesions of the substantia innominata on attentional function in the rat: Further implications for the role of the cholinergic neurons of the nucleus basalis in cognitive processes. $B e$ havioural Brain Research, 35, 221-240.

Sánchez-Santed, F., de Bruin, J. P. C., Heinsbroek, R. P. W., \& VerWER, R. W. H. (1997). Spatial delayed alternation of rats in a T-maze: Effects of neurotoxic lesions of the medial prefrontal cortex and of T-maze rotations. Behavioural Brain Research, 84, 73-79.
Sarter, M., Bruon, J. P., Givens, B., Moore, H., McGaughy, J., \& MCMAHON, K. (1996). Neuronal mechanisms mediating druginduced cognition enhancement: Cognitive activity as a necessary intervening variable. Cognitive Brain Research, 3, 329-343.

SATOH, K., \& Fibiger, H. C. (1986). Cholinergic neurons of the laterodorsal tegmental nucleus: Efferent and afferent connections. Journal of Comparative Neurology, 253, 277-302.

Seamans, J. K., Floresco, S. B., \& Phillips, A. G. (1995). Functional differences between the prelimbic and anterior cingulate regions of the rat prefrontal cortex. Behavioral Neuroscience, 109, 1063-1073.

Seamans, J. K., Floresco, S. B., \& Phillips, A. G. (1998). D receptor modulation of hippocampal-prefrontal cortical circuits integrating spatial memory with executive functions in the rat. Journal of Neuroscience, 18, 1613-1621.

Shallice, T., \& Burgess, P. (1991). Higher-order cognitive impairments and frontal lobe lesions in man. In H. S. Levin, H. M. Eisenberg, \& A. L. Benton (Eds.), Frontal lobe function and dysfunction (pp. 125-138). New York: Oxford University Press.

Silva, M. G., Boyle, M. A., Finger, S., Numan, B., Bouzrara, A. A., \& ALMI, C. R. (1986). Behavioral effects of large and small lesions of the rat medial frontal cortex. Experimental Brain Research, 65, 176-181.

Sokolowski, J. D., \& Salamone, J. D. (1994). Effects of dopamine in the medial prefrontal cortex on DRL performance and motor activity in the rat. Brain Research, 642, 20-28.

Stoehr, J. D., Mobley, S. L., Roice, D., Brooks, R., Baker, L. M., WILEY, R. G., \& WENK, G. L. (1997). The effects of selective cholinergic basal forebrain lesions and aging upon expectancy in the rat. Neurobiology of Learning \& Memory, 67, 214-227.

Stuss, D. T., \& BENSon, D. F. (1986). The frontal lobes. New York: Raven.

Swain, S. A., Polkey, C. E., Bullock, P., \& Morris, R. G. (1998). Recognition memory and memory for order in script-based stories following frontal lobe excisions. Cortex, 34, 25-45.

TURCHI, J., \& SARTER, M. (1997). Cortical acetylcholine and processing capacity: Effects of cortical cholinergic deafferentation on crossmodal divided attention in rats. Cognitive Brain Research, 6, 147 158.

Uylings, H. B., \& VAN EDEN, C. G. (1990). Qualitative and quantitative comparison of the prefrontal cortex in rat and in primates, including humans. Progress in Brain Research, 85, $31-62$.

Wilkinson, L. S., Humby, T., Killcross, A. S., Torres, E. M., Everitt, B. J., \& Robbins, T. W. (1998). Dissociations in dopamine release in medial prefrontal cortex and ventral striatum during the acquisition and extinction of classical aversive conditioning in the rat. European Journal of Neuroscience, 10, 1019-1026.

Williams, J. M., Mohler, E. C., \& Givens, B. (1999). The role of the medial prefrontal cortex in attention: Altering predictability of task difficulty. Psychobiology, 27, 462-469.

Wilson, F. A., Scalaidhe, S. P. O., \& Goldman-Rakic, P. S. (1993). Dissociation of object and spatial processing domains in primate prefrontal cortex. Science, 260, 1955-1958.

WoOLF, N. J. (1991). Cholinergic systems in mammalian brain and spinal cord. Progress in Neurobiology, 37, 475-524.

YANG, C. R., \& MOGENSON, G. J. (1990). Dopaminergic modulation of cholinergic responses in rat medial prefrontal cortex: An electrophysiological study. Brain Research, 524, 271-281.

ZahrT, J., Taylor, J. R., Mathew, R. G., \& Arnsten, A. F. T. (1997). Supranormal stimulation of $D_{1}$ dopamine receptors in the rodent prefrontal cortex impairs spatial working memory performance. Journal of Neuroscience, 17, 8528-8535.

(Manuscript received September 8, 1999; revision accepted for publication February 29, 2000.) 